\title{
The Existence of Two Types of Colloidal Solutions of Molecules Fullerene $\mathbf{C}_{60}$
}

\author{
D.V. Schur ${ }^{1, *}$, S. Yu. Zaginaichenko ${ }^{1}$, T.N. Veziroglu ${ }^{2}$, A. Veziroglu ${ }^{2}$ and M.T. Gabdullin ${ }^{3}$ \\ ${ }^{1}$ Frantsevich Institute for Problems of Materials Science of NASU, 3, Krzhyzhanovsky str., Kiev 03142, \\ Ukraine \\ ${ }^{2}$ University of Miami, International Association for Hydrogen Energy, 5794 SW 40 St. \#303, Miami, FL 33155, \\ USA \\ ${ }^{3}$ National Nanotechnology Laboratory, al-Farabi Kazakh National University, 71 al-Farabi str, Almaty 050040, \\ Kazakhstan
}

\begin{abstract}
The last thirty years scientists carried out an active search of universal parameter of solvent C60 for predicting the solubility of fullerenes. However, this parameter was not found up to these days. In this paper it has been found an explanation of the impossibility of detection of such parameter.

In present paper the features of the solubility of fullerene C60 molecules in nonpolar solvents have been studied. The molecule state diagram of the fullerene C60 molecules in solution at temperatures $265 \div 308 \mathrm{~K}$ and pressure range $1 \div 100$ $\mathrm{MPa}$, indicating the existence field of $\beta$ - and $\gamma$-modifications, has been constructed.

Moreover, it has been shown that in the solutions of fullerenes $\mathrm{C} 60$ in the $\mathrm{y}$-state the solubility decreases with a rise in temperature since the chemical activity of fullerene molecule increases. For this reason during extraction procedure the temperature rise causes the value of extraction rate to increase, but solubility - to decrease, i.e. to diminish the concentration of fullerenes molecules in the solvent volume.
\end{abstract}

Keywords: Fullerene $\mathrm{C}_{60}$ molecule, molecule diagram, colloidal solution, solubility isobar, solutions classification.

\section{INTRODUCTION}

Constant research hydrogen capacity of synthesized nanostructured carbon materials [1-7] made experimental researchers to solve problems of the effective methods development for the extraction of fullerenes from fullerene-containing soot. Today, a large number of solvents have been studied, and a large database of experimental data on the solubility of C60 in solvents belonging to different classes of chemical compounds has been accumulated. The search for a universal solubility parameter $\mathrm{C} 60$ was the target of the first studies on the solubility of fullerenes. In most cases the $\mathrm{C} 60$ dissolution process was considered within the rule "like dissolves like". Various physical and chemical properties of substances were considered as the similarity factor. It was found that none of the solvents physical properties which studied is adequate. Authors of some works have suggested that fullerenes and other nanostructures can hardly be presented with the "classical" molecular circuit.

In some works searches of quantitative dependence of the solubility of C60 fullerene molecules from the solvent structure in solution were carried out. If predictive parameters for some compounds were

*Correspondence Address to this author at the Frantsevich Institute for Problems of Materials Science of NASU, 3, Krzhyzhanovsky str., Kiev 03142, Ukraine; Tel/Fax: +380 (044) 324 0381; E-mail: dmitry.schur@gmail.com found, however, they were not detected for the aliphatic and aromatic compounds which are powerful C60 solvents. We had found a pattern of influence groups, substituting hydrogen in polimetilbenzene molecule, on the solubility of $\mathrm{C60}$ [8]. The analysis of data on the C60 fullerene molecule the solubility showed that the rule "like dissolves like" does not always work. Thus, according to this hypothesis, the maximum C60 solubility is observed in aromatic hydrocarbons. However, there are other examples. These include some non-aromatic solvents such as CS2, halogenated alkanes, where $\mathrm{C60}$ solubility is higher than in benzene.

In our view, the complex behavior of the $\mathrm{C} 60$ fullerene molecule in solution is determined by its duality. The dualism of the C60 fullerene molecule is manifested in that, it being a molecule, exhibits the properties of colloidal particles in solutions at temperatures above $273 \mathrm{~K}$. In this paper an attempt is made to classify solutions C60 depending on the state of the fullerene molecule in solution.

\section{Solutions of C60 Fullerene Molecules}

According to our ideas set out in [9-14] and above, there are three types of solutions C60: 1. true; 2 . I- type colloidal solution; 3 . Il-type colloidal solution. On the formation of a particular type of solution is affected as 


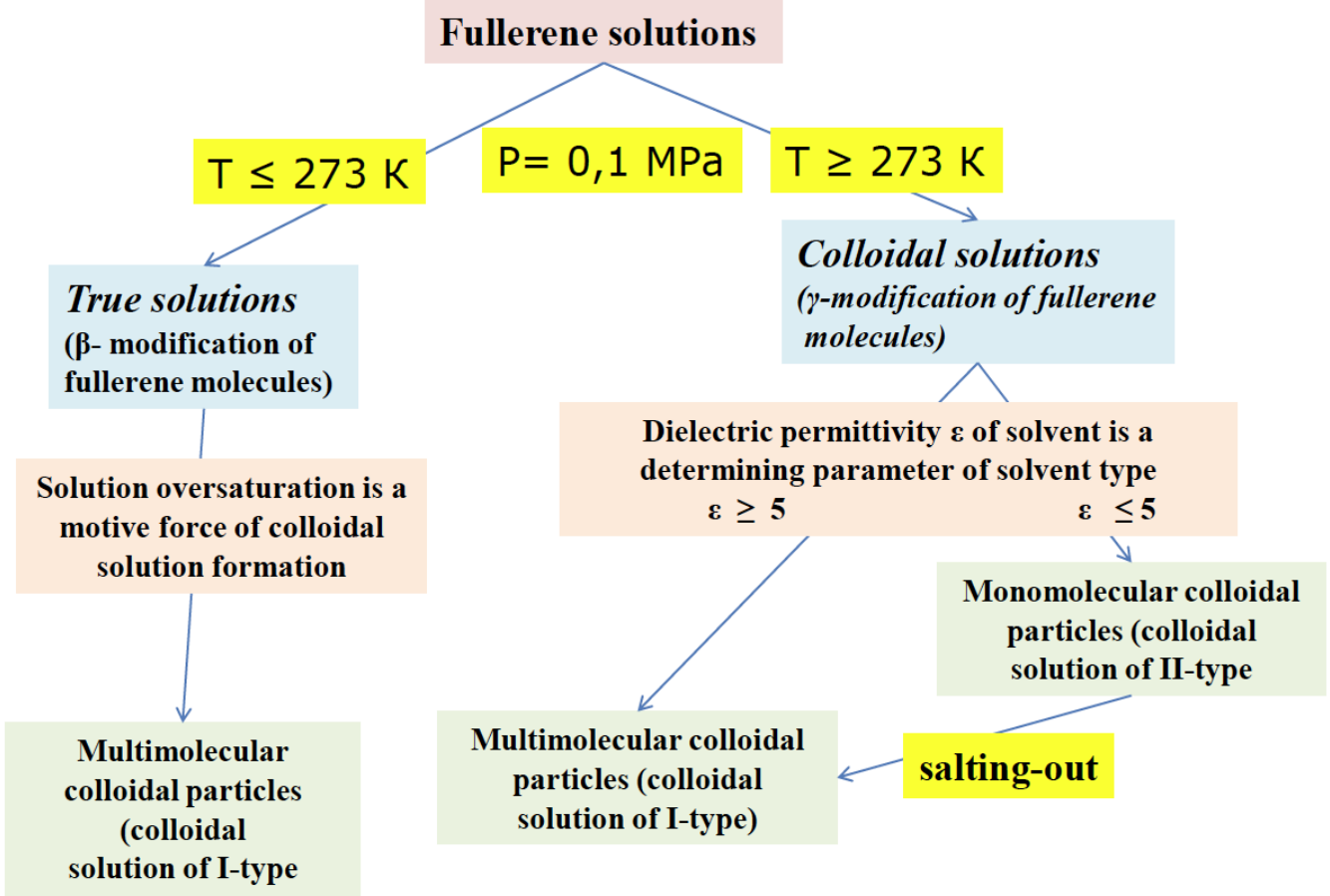

Figure 1: The schematic diagram and mechanism of formation of fullerenes molecules.

the state of the fullerene molecule (i.e. $\beta$ - or $\gamma$ modification [9-12]), depending on the thermodynamics of the system of the existence, well as nature of the solvent [8, 12-15], an important indicator which is the dielectric constant of the solvent itself (Figure 1).

Considering thermodynamical conditions of the solutions existence, it should be noted that under normal pressure boundary of the existence of $\beta$ - and $y$ modification of fullerene molecules (phase) is the temperature of $273 \mathrm{~K}[9,10,12]$. Below this temperature, there is a modification of the $\beta$ - of fullerene molecules, and above - $\gamma$ - modification of the fullerene molecules.

The $\beta$-state (below $T=273 \mathrm{~K}$ ) fullerene molecules dissolved in a liquid forms simple (molecular) solutions. In the case of solution oversaturation fullerene molecule coagulates and forms a colloidal solution of Itype, consisting of nano-sized crystals of fullerene and solvent molecules.

It should be noted that $\mathrm{Y}$-state of fullerene molecules is characterized by formation of colloidal solutions only, which differ in the amount of fullerene molecules in colloidal particles that formed.

Since the solutions, formed as a result of coagulation of fullerene molecules, i.e. the solutions in which the colloidal particles are combined by two or more molecules of fullerenes, are the colloidal solutions of I-type, then the systems, produced by the colloidal particles formed by one fullerene molecule, should be related to the colloidal solution of II-type.

Considering solutions of fullerene molecules from position the nature of the solvent impacting on solubility, it should be noted that the nature of the solvent effects on the mechanism of interaction of solvent molecules with molecules of fullerene. The difference in the mechanisms of interaction between solvent molecules and fullerene molecules leads to formation of two types of colloidal fullerene solutions.

If we consider the colloidal solutions of both types, it must be emphasized that the colloidal solutions of Itype make the heterogeneous system. The solutions of II-type can be identified as a homogeneous system as they are in the $\beta$-state. However it is pertinent to note that, by virtue of the fact that the process of micelleformation goes on the surface of fullerene molecules, this system may be thought of as the heterogeneous. In this manner the fullerene molecules in the $y$-state represents the colloidal particle making micelle, formed by the fullerene molecule surface and adsorbed layer and it constitutes the phase differing from the aggregate state both of solid and of liquid.

In the subsequent subsections of paper present the results of experimental investigations substantiating the conception of existence of fullerene $\mathrm{C} 60$ molecules in solutions of three types. 


\section{Anomalous Solubility of Fullerene Molecules}

Notification of American scientists about the anomalous solubility of the fullerene molecules appeared in 1993 [16]. It reported the experimental results on the temperature dependence of solubility of $\mathrm{C}_{60}$ molecules. They observed that this dependence displays the anomalous behavior on the experimental curves in the form of solubility maximum at the temperature near $280 \mathrm{~K}$ in all presented organic solvents. The revealed dependence did not fit into existing notion of solubility of compounds in the organic solvents. The solubility increase with the rising temperature is considered to be normally regular. However, neither this investigation nor works of next twenty years did not answer to the questions being examined.

Later the solubility of fullerene molecules $\mathrm{C}_{60}$ was studied at temperatures of 278,2-308,2 $\mathrm{K}$ and pressures up to $340 \mathrm{MPa}$ [17]. The $\mathrm{C}_{60}$ solubility initially increases with increasing pressure and then decreases with a sharp peak indicating on the analogy in connection with the solid-phase transformation. The thermodynamic analysis of solubility, conducted in this paper, has confirmed the assumption that two solid phases, corresponding to $\mathrm{C}_{60}$ (fcc) and $\mathrm{C}_{60} \cdot 2 \mathrm{C}_{6} \mathrm{H}_{5} \mathrm{CH}_{3}$ solvates, exist in the solution. The authors have pointed out an unusual phenomenon of $\mathrm{C}_{60}$ solubility enhancement with increasing pressure in a lowpressure region, when compared with the decrease in solubility of the nonpolar molecular solids generally observed with rises in pressure.

The answer to both questions has been given in the papers [11, 12] and discussed in paper [9]. The careful experimental study of the temperature dependence of the $\mathrm{C}_{60}$ solubility in toluene has been conducted to ascertain the anomalous behavior of fullerene molecules in solutions. We have carried out three series of experiments. The plot of the temperature dependence of the $\mathrm{C}_{60}$ solubility in toluene (Figure 2 ) [12] has been constructed on the basis of the arithmetic average values obtained in three series of experiments.

The presented results qualitatively confirm the data received by other researchers $[16,17]$. It is evident from experimental data that the solubility of fullerene molecules with increasing temperature of the solution in the initial section (the portion DB of the curve) obeys the laws of behavior of the true solutions, but at a higher-temperature portion BA it behaves abnormally. Based on the proposed in $[9,18,19]$ temperature ranges of existence of the fullerene molecules

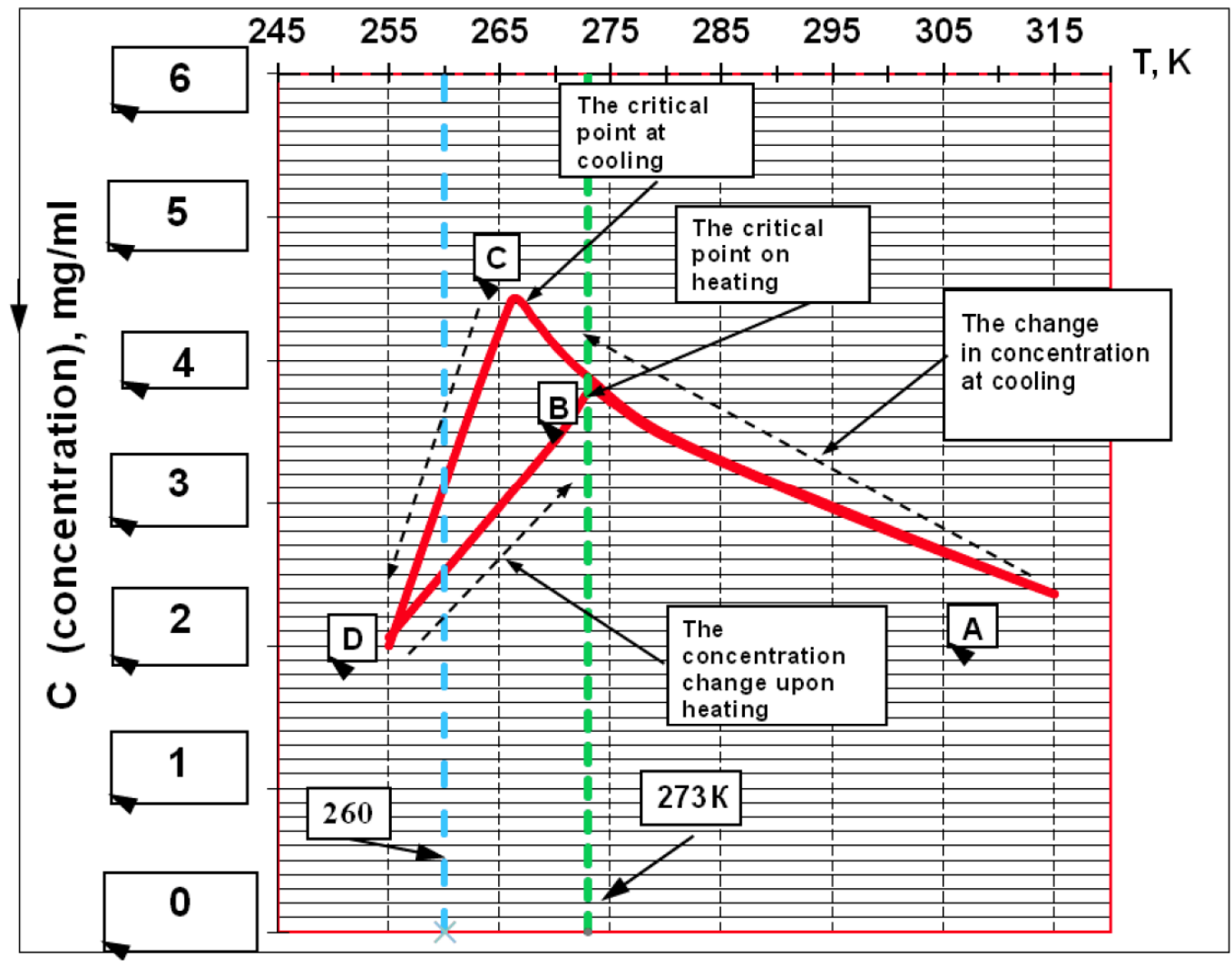

Figure 2: The concentration hysteresis of solubility of fullerene $C_{60}$ under changes of temperature [12]. 
modifications, we can assume that $\beta$-modification exists in solution at the portion DB, and $\gamma$-modification at the portion BA.

If we correct the family of isotherms of solubility of fullerene molecules constructed according to the data of Sawamura S. paper [17], and represent them as solubility isobars and with the use of updated our data on the isobars $(15,65$ and $80 \mathrm{MPa})$ as well as research data (Figure 2) found at the atmospheric pressure, then we obtain a family of isobars plots for solubility of fullerene molecules in toluene, presented in Figure 3.

It is believed that maxima on the curves of the isobars of fullerene molecules solubility correspond to the transition of $\beta$-modification into $\gamma$-modification, and we have drawn the straight line through the points of molecular transitions on the isobars. Then, we transfer the tangent points of these maxima and straight line $A B$ (Figure 2) to the P-T diagram and get the state diagram of the fullerene molecules in solution (Figure 3 ).

The field enclosed by the temperature axis and the curve $A B$ shows the thermodynamic conditions corresponding to the existence of fullerene molecules in the $y$-modification. The field between the curve $A B$ and the pressure axis corresponds to the thermodynamic conditions of existence of $\beta$ modification of fullerene molecules, the curve $A B$ shows the line of transition between the molecular modifications. Hence, it is evident from the phase diagram of the fullerene molecules in solution in the temperatures interval of $265-308 \mathrm{~K}$ and pressures range of 0.1 - $100 \mathrm{MPa}$ that for the transfer of fullerene molecule into the $\beta$-state at the room temperature there is a need to apply to the system the external pressure more than $70 \mathrm{MPa}$.

For this reason we can also conclude that all studies, carried out by various researchers with fullerene molecules at the temperature higher $273 \mathrm{~K}$, were directed to investigate the properties of $\mathrm{Y}$ modification only.

On the assumption that the true solution is formed on the solubility curve in the $\beta$-field, but after the concentration maximum the fullerene-containing complexes or the colloidal solutions of II-type are formed in the form of $Y$-modifications it can be somewhat in another way to interpret the experimental results on the Figure 2. In particular, it is possible to assume that the concentration hysteresis appears by virtue of the geometrical dimensions of the fullerenecontaining complexes to reduce on the curve portion "AB" with the reducing temperature and decrease in the chemical activity of the fullerene molecules (at the expense of decrease of the amount of solvent molecules in the complex). As this takes place, an additional amount of the solvent molecules, involved further in the moving of the additional quantity of

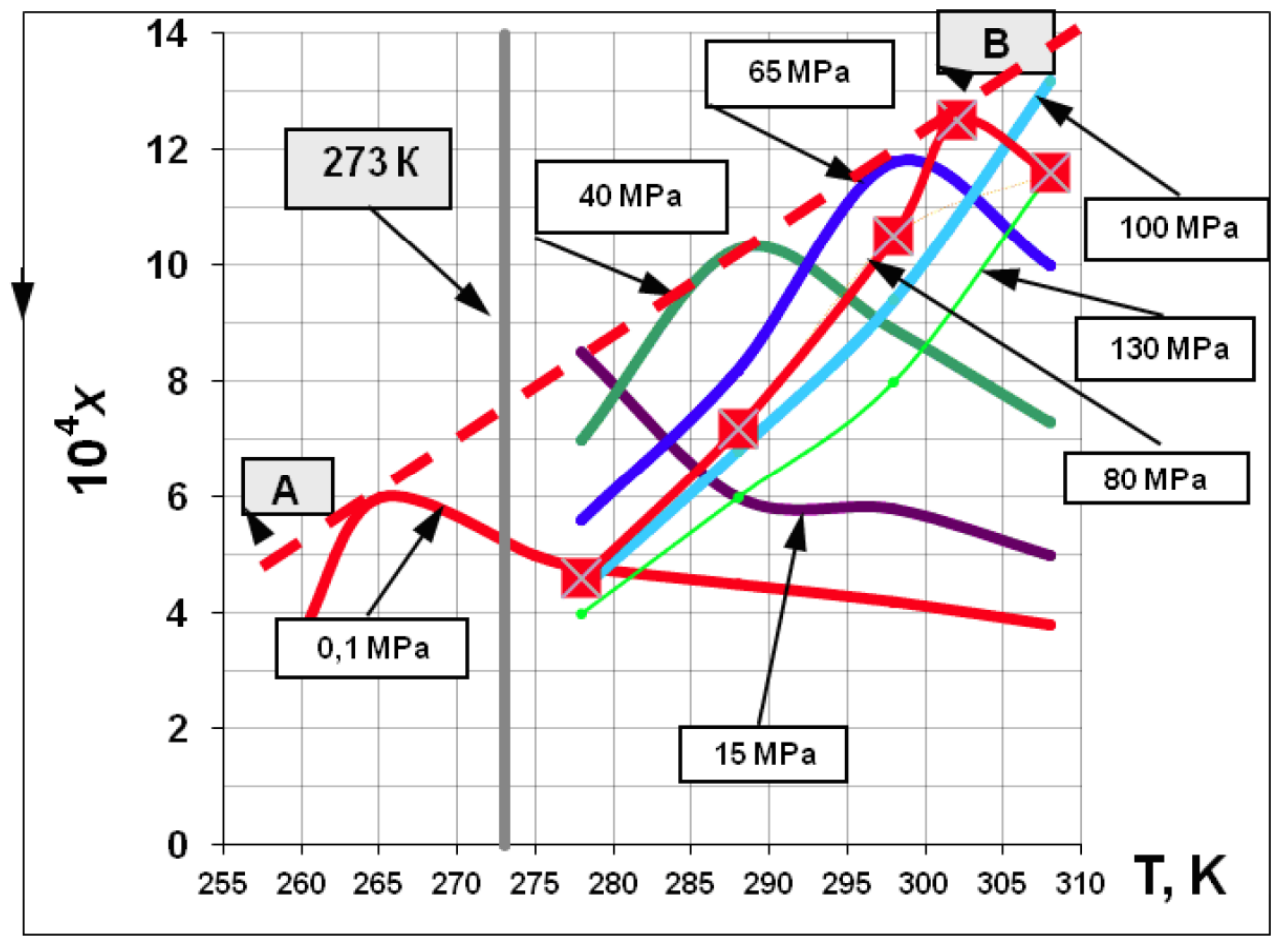

Figure 3: The isobars of solubility of fullerene $C_{60}$ molecule in toluene [12]. 
fullerene molecules from fullerite to the solution, is released. In this case the temperatures of complexes formation exceed the temperatures of their destruction.

Based on this, we can conclude that on the curve section "BC" (Figure 2) the structure changing of the fullerene molecules occurs, and consequently of the complexes. With decrease in temperature, the dissolved molecules are still confined by the solvent molecules in the compound of "depleted" complexes, whereas the fullerene molecules of $\beta$-state, being in a less active form and binding the smaller amount of solvent molecules, again dissolve and form the true solution. The existence of two systems in the solution on the curve portion BC produces the excess concentration of fullerenes in the solution. The migration of critical point (the concentration maximum) in the curve portion BC leads that different authors suggest the presence of maximum of dissolution at different temperatures in the range from 260 to $280 \mathrm{~K}$. In addition the solvent nature, defined by the force of interaction of first layer of solvent molecules with the fullerene molecule, can affect on the value of induced period BC. Point " $\mathrm{C}$ " corresponds to the critical temperature of the existence of the complexes. Below this temperature, the complexes are completely destroyed, and the system forms a true solution. Locating of the system in the certain point of concentration hysteresis is defined by the state of the fullerene molecule in the solution. The temperature of molecular transition can be moved on a scale of temperatures depending on the chemical activity of the solvent and the system pressure. Based on the experimental data we can conclude that of prime importance is the knowledge of the sequence of measurements for the interpretation of the effects of the temperature dependence of the fullerene solubility.

It has been also shown in paper [9] that increasing pressure at the dissolution of fullerene molecules stabilizes the $\beta$-state, making difficult their transition to the $y$-state. For this reason, the critical point on a scale of solubility moves above (Figure 2), i.e. with rising pressure the transition shifts to a more hightemperature region (Figure 4). Since in the $\beta$-state the fullerene solution in toluene behaves as true - that is the solubility increases with the rising temperature then the shift of transition to the high-temperature region tend to increase the upper temperature boundary of $\beta$-state existence and cause solubility to increase. This provides an explanation for the observed anomalies in the paper [17].

Thus, we have constructed the state diagram of fullerene molecules, corresponding to their behavior in the dissolved state. It has been shown that two

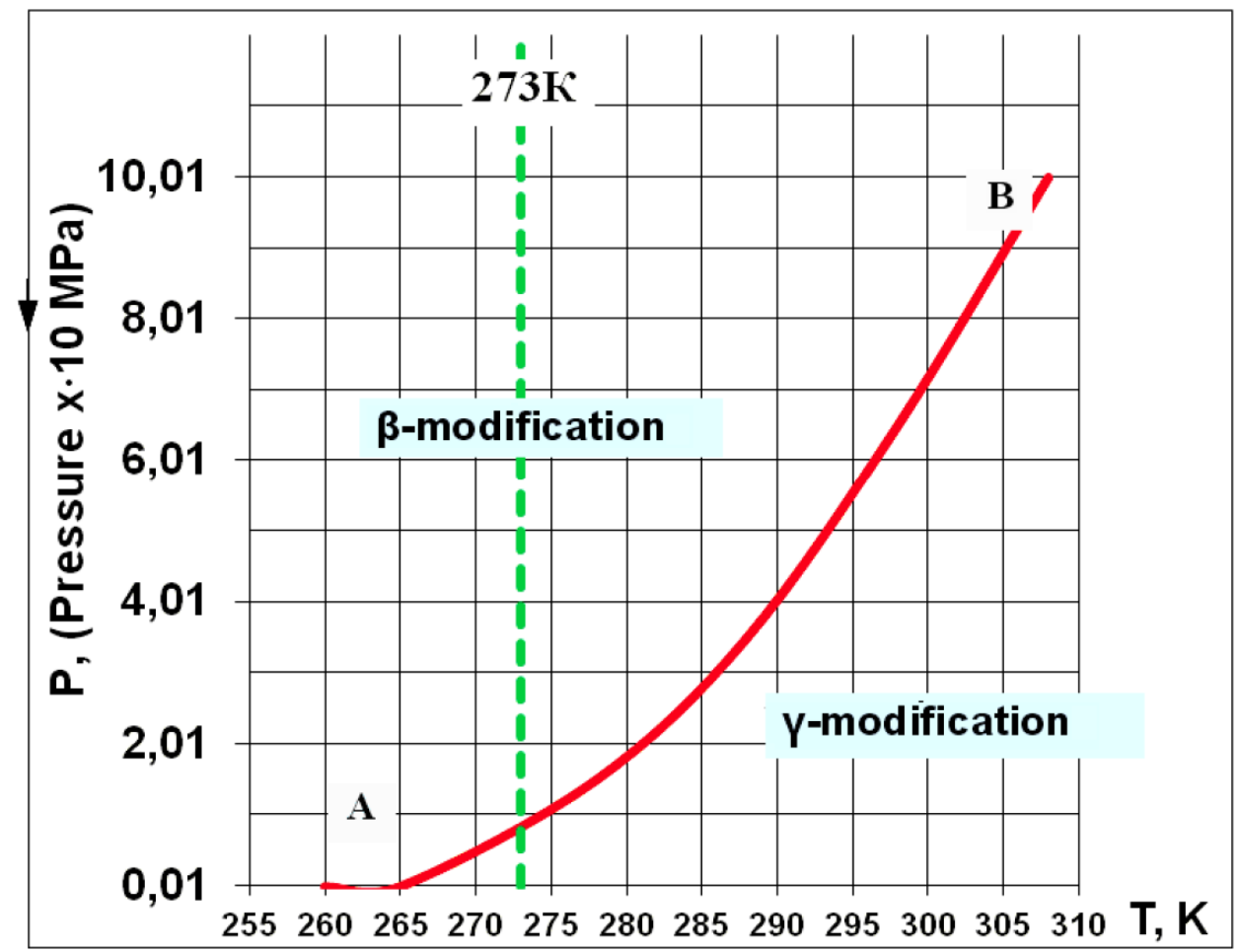

Figure 4: The phase diagram of the state of fullerene $\mathrm{C}_{60}$ molecule in solution at temperatures $265 \div 308 \mathrm{~K}$ and in the pressure range $1 \div 100 \mathrm{MPa}$ indicating the existence field of $\beta$ and $\mathrm{\gamma}-$ modifications [12]. 
different processes affect on the properties of solutions of fullerene molecules, as the process of restructuring of fullerene molecules, which entails the change of mechanism of interaction between molecules of fullerenes and solvent molecules. This initiates the process of transition of the true solution into the colloidal and reversely. Both of them are determined by the system thermodynamics.

It should be noted an interesting feature of the considered process of transformations in the $\mathrm{C}_{60}$ molecule, which is in solution. Its temperature is near to the critical temperature of existence of the living nature $-0^{\circ} \mathrm{C}$.

Also, in support that the concentration hysteresis is dictated by the formation of the fullerene-toluene complexes in $Y$-solution and in verification that the solvent molecules act as stabilizers in $\gamma$-solutions of fullerenes, we have conducted the research of processes of electrophoresis, the salting-out at low temperatures and photopolymerization of fullerenes solutions in toluene.

\section{Complexes with Charge-Transfer}

Thus, it has been shown that the fullerene molecule in solutions at temperatures above $250 \mathrm{~K}$ (depending on the temperature) exists in the two states, as $\beta$ and $\mathrm{\gamma}$. It has been assumed that the true solution of fullerene molecules is formed in the $\beta$-state, and the fullerene-toluene complexes are formed by molecules in the $\mathrm{y}$-state.

To confirm this assumption, the experimental studies of the fullerenes solubility in the monosubstituted of benzene have been carried out (Table 1) [13-15]. The fullerenes solubility in benzene is $1.5 \mathrm{~g} / \mathrm{l}$.

The experiments have shown that by adding of an electron-donating substituents to the benzene the fullerenes solubility is increased, and by attaching of an electron-acceptor substituents to the benzene the solubility of fullerenes in them is reduced as compared with benzene. The obtained data demonstrates evidently that at the temperature above $270 \mathrm{~K}$ the fullerene molecules in solutions form the chargetransfer complexes (CTC).

\section{Electroneutral Toluene-Fullerene Complexes (II- Type Colloidal Solutions)}

To confirm the hypothesis of the existence of fullerene in solutions as electrically neutral fullerenetoluene micelle or complexes experimental studies by electrophoresis of fullerene solution in toluene were conducted. Four systems were considered: toluene, fullerene solution in toluene, toluene and ethanol, fullerene solution in toluene - ethanol mixture. Experiments have shown that no toluene or toluene solutions of fullerenes are conductors of electric current at voltage to $2000 \mathrm{~V}$ and $3-5 \mathrm{~mm}$ distance between electrodes. However, when adding ethanol in toluene electric current is appeared which intensity depends on the amount of ethanol and increases with the voltage rising on the electrodes.

A similar situation exists with the addition of ethanol to a solution of fullerene in toluene (Figure 5). This behavior could be attributed also by the presence of ethanol in the solution, but in this case, fullerite

Table 1: The Donor-Acceptor Mechanism of Fullerene $\mathrm{C}_{60}$ Dissolution in Aromatic Hydrocarbons (Benzene Monosubstituted)

\begin{tabular}{|c|c|c|c|c|}
\hline \multirow{2}{*}{ Electron-donor substituents } & \multicolumn{2}{|c|}{ Composition of formed isomers, \% } & \multirow{2}{*}{ Solubility of fullerene $\mathbf{C}_{60}, \boldsymbol{m g} / \boldsymbol{m l}$} \\
\cline { 2 - 5 } & ortho- & meta- & para- & \\
\hline \hline Chlorobenzene $\left(\mathrm{C}_{6} \mathrm{H}_{5} \mathrm{Cl}\right)$ & 29,6 & 0,9 & 69,5 & 5,70 \\
\hline Toluene $\left(\mathrm{C}_{6} \mathrm{H}_{5} \mathrm{CH}_{3}\right)$ & 58,5 & 4,4 & 37,1 & 2,90 \\
\hline Ethylbenzene $\left(\mathrm{C}_{6} \mathrm{H}_{5} \mathrm{CH}_{2} \mathrm{CH}_{3}\right)$ & 45,0 & 6,5 & 48,5 & 2,60 \\
\hline
\end{tabular}

\begin{tabular}{|c|c|c|c|c|}
\hline \multirow{2}{*}{ Electron-acceptor substituents } & \multicolumn{2}{|c|}{ Composition of formed isomers, $\%$} & \multirow{2}{*}{ Solubility of fullerene $\mathbf{C}_{60}, \boldsymbol{m g} / \boldsymbol{m l}$} \\
\cline { 2 - 5 } & ortho- & meta- & para- & 0,80 \\
\hline \hline Nitrobenzene $\left(\mathrm{C}_{6} \mathrm{H}_{5} \mathrm{NO}_{2}\right)$ & 6,4 & 93,3 & 0,3 & 0,42 \\
\hline Benzaldehyde $\left(\mathrm{C}_{6} \mathrm{H}_{5} \mathrm{COH}\right)$ & 19,0 & 72 & 9,0 & 0,41 \\
\hline Benzonitride $\left(\mathrm{C}_{6} \mathrm{H}_{5} \mathrm{CN}\right)$ & 17 & 81 & 2,0 & 0,0 \\
\hline
\end{tabular}




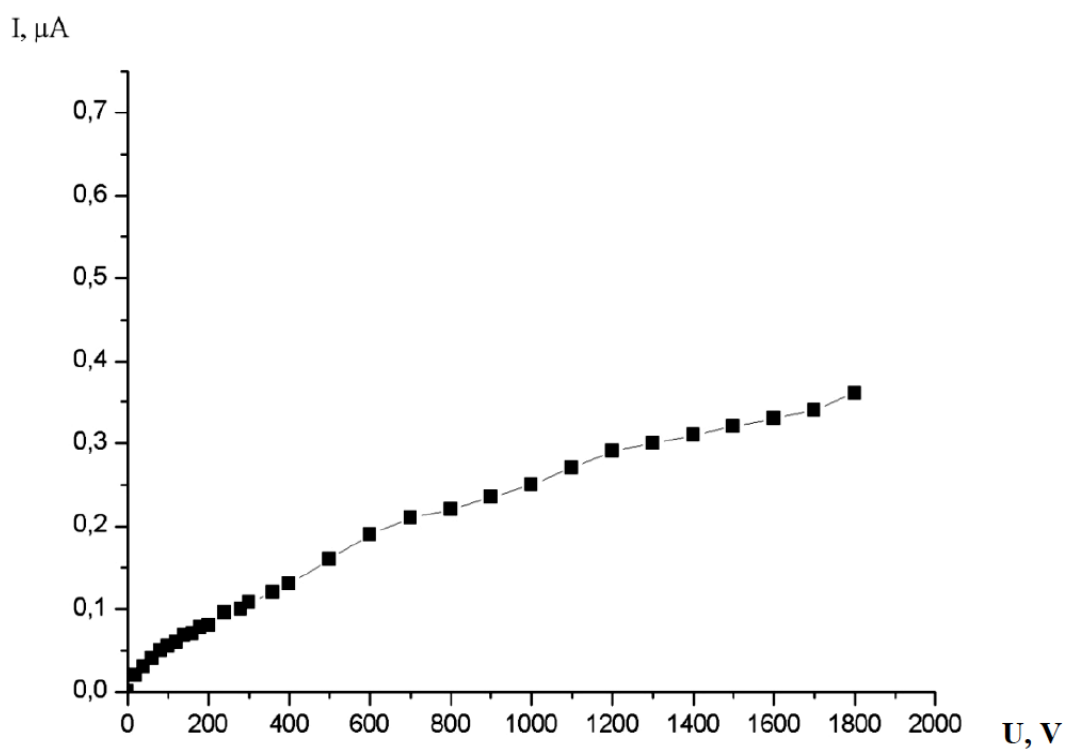

Figure 5: The dependence of the electric current I in saturated solution of fullerenes in toluene with addition of ethanol on the voltage $U$ between electrodes.

crystallizes on the positive electrode. In the absence of an electric field fullerite it precipitates as deposit. This can be explained by the fact that, since ethanol molecules do not react with fullerene molecules, then adding ethanol forms a solution of alcohol molecules with molecules of toluene (azeotrope) and destroy solvent shell of a micelle, existing in solution.

Moreover, it shows that the fullerene micelles in solution after partial loss of solvent molecules from the shell, has got a negative charge and deposit formed as crystals on the positive electrode under the influence of an electric field. This indicates the presence of a negative potential on the outside shell of the molecule, i.e. uneven distribution (under the influence of solvent molecules) m-electron density at the inner and outer surfaces of the carcass of the fullerene molecule. It also follows that the core of the complex (colloidal particle) is negatively charged [20]. The experiment proves the existence in solutions of the electrically neutral fullerene-type colloidal toluene complexes in the form of micelles.

\section{Effect of the Fullerene Molecules State on the Photopolymerization Process}

To support the above assumptions experimental studies were carried out using photopolymerization of gamma-fullerene solutions as a confirmation that the solvent molecules are stabilizers of fullerene-toluene complexes.

The phenomenon of photopolymerization of fullerenes solutions was observed during long-term storage solutions in the daytime, when the purple color of the solution changed to yellow. Our experiment was the fact that the saturated toluene solution of fullerenes (with excess of fullerite in flask) was irradiated using a flash.

The experiments have shown that when temperature of a freshly prepared solution decreases from 307 to $283 \mathrm{~K}$ and they are subjected to flash irradiation fullerene is polymerized, and the solution changes its color to pale yellow. Apparently micelles are destroyed upon irradiation, and the fullerene molecules interact each other to form a polymer.

At the time of photopolymerization, many solvent molecules are released, which going in interaction with fullerite, would again form the charge-transfer complexes, in this case restoring the color of the solution. However, the mentioned process does not take place; this confirms formation of complexes on a basis of polymer. The stabilized polymeric molecules by the solvent molecules provide yellowish color to a solution. By adding ethanol to the solution the fullerenes are salting out.

Thus, the experiment has shown that both in the solutions of polymers and in the fullerenes solutions the solvent molecules act in the role of stabilizers of colloidal particles, as well as regarding the irradiation moment the fullerene molecules are present in a solution in the $y$-state in the form of micelles.

At temperatures below $273 \mathrm{~K}$, the fullerene $\mathrm{C}_{60}$ molecules under the influence of irradiation does not 
polymerized, and in polymers the depolymerization process takes place [21-24], that confirms the low chemical activity of fullerene molecules in the $\beta$-state.

In addition, the same color both of the $\mathrm{C}_{60}$ solution in $\beta$ - and in $\gamma$-state indicates that in both modifications the doubled pentagons manifest themselves as chemically active center in the frame of fullerene $C_{60}$ molecules and the chemical activity of fullerene molecules is regulated by the outside component of $\pi$ electron cloud of the frame.

Experimentally, we have proved that in $\mathrm{y}$-solutions of fullerenes, the solvent molecules act as stabilizers of colloidal particles.

\section{The Colloidal Solutions of II-Type}

As the solvent molecules act as stabilizers in toluene-fullerene complexes, such solutions may be called colloidal.

This conclusion can be supported by consideration of the geometric parameters of the fullerene molecule [9]. Dimensional and boundary parameters can be attributed to them.

1. The size parameter. In view of the fact that the Van-der-Waals diameter of fullerene C60 molecule (more than $1 \mathrm{~nm}$ ) is at the border between the extremely high-dispersed and molecular-ionic systems and the molecular size of fullerene compounds in the solution exceeds this limits, then on this parameter the fullerene C60 molecules are located in the dimensional region of extremely high-dispersed systems and fall into the size range of colloidal particles.

2. Boundary parameter. The colloidal state of matter - a special state characterized by a high ratio of surface atoms of the colloidal particles to the number of atoms in the volume of the particle. Fullerene molecule - a limit point of the state in which the above ratio tends to infinity. For this reason, the main parameter that transfers the $\mathrm{C} 60$ fullerene molecule into the category of colloidal particles is presence the surface of the fullerene molecule, separating it from the external environment. The specific surface area in the molecular systems such as true (molecular-ionic) solutions is absent, because of a molecule does not have the surface in the usual sense of the word. On the other hand, the surface of the fullerene molecule cannot be regarded as the surface of a regular solid-state because it does not have the subsurface atoms.

Thus, the feature of the fullerene molecule structure (and therefore their properties) is that it can be considered as a special colloidal particle that has the surface atoms only and does not have bulk ones. The density of the m-electron cloud of fullerene molecules, similar to carbon nanotubes, is distributed on the surface of the particles, and in its entirety. Redistribution of density may vary depending on the external environment, as evidenced by the formation of endofullerenes. Redistribution density $\pi$-electron cloud within the scope and outside of the fullerene molecules enables them to exhibit the properties in solution as the molecules well as the colloidal particles. The first state of molecules can be called $\beta$-state, and the second $-\gamma$ state of fullerene molecule.

The surface of one gram molecule of $\mathrm{C} 60$ is equal to $2628 \mathrm{~m} 2$. Very high surface energy of the fullerene molecules makes them active colloidal particles.

The volume of toluene-fullerene complex formed in solution, can reach $398 \mathrm{~nm} 3$. Thus, the diameter of the micelles formed by dissolving $\mathrm{y}-\mathrm{C} 60$ in toluene at room temperature and atmospheric pressure is in the range $8 \div 9 \mathrm{~nm}$.

Thus, the dualism of fullerene molecule allows it to form colloidal solutions with the formation of micelles.

\section{The Mechanism of Solvent Molecules Interaction with Molecules of Fullerene C60}

Now we have to consider the causes of the low solubility of $\mathrm{C} 60$ in a solvent, as a consequence of the colloidal nature of the $y$-solutions. In addition, we will answer the question of why not all of the liquids are able to dissolve fullerene.

We assume that the process of dissolution of the C60 fullerene molecules at the formation of colloidal solutions of II-type occurs in the following way. As a result of the interaction of solvent molecules with the fullerene molecules, which leads to the formation of charge-transfer complexes, there is a redistribution of intermolecular electron density, which entails its intramolecular redistribution between the atoms forming the two interacting molecules. In this case, the electron density and hence the reactivity of the solvent molecules that had interacted will differ from the original molecules. 
The solvent molecule formed the complex with fullerene molecule, will be able to enter into interaction with the free solvent molecule, but just in the new conditions because it will has the properties of the formed complex and carry the influence of fullerene molecule.

The interaction is balanced in the case when of electron-deficient properties of the fullerene molecule will be offset by m-electron-donor properties of the solvent molecules. In such the analysis of the interaction process it is logical to expect that the greater the donor ability of the solvent molecule, the smaller its molecules must be used to saturate the electronic deficiency of the fullerene molecule.

In this case, when $\mathrm{T}$ is above $273 \mathrm{~K}$, the fullerene molecules are converted into the colloidal particles and form around themselves the adsorption layer of solvent molecules. To compensate for the acquired potential around colloidal particle the external mobile adsorption layer of the solvent molecules is formed, transforming the colloidal particle into micelle. Based on studies of the diffusion mobility of the fullerene molecule in solution, we can conclude that in such solutions colloidal particles diffuse only. The lifetime of the micelle is very small in connection with mobility of molecules of the outer layer [9].

When forming a colloidal solution by the C60 fullerene molecule, the diameter of colloidal particle (depending on the nature of the solvent) can be increased to $5 \mathrm{~nm}$, whereas a diameter of the micelles can be $30 \mathrm{~nm}$ or more [12].

Based on the experimental data (Table 2), it can be concluded that with an increase in the solubility of the fullerene (i.e. chemical activity of solvent molecules for fullerene) the thickness of the sorption layer decreases.

The minimum distance between the centers of two neighboring micelles can reach $6.5 \mathrm{~nm}$, and the minimum thickness of the barrier between the fullerene molecules can be $5.5 \mathrm{~nm}$. This space is filled with solvent molecules of adsorbed layer constituting the dispersion medium and acting as a stabilizer of fullerene molecules in solution. They hold the fullerene molecule at a desired distance, creating an energy barrier of anticoagulation. Reducing of the energy barrier, which protects the fullerene molecules from sticking together under the influence of molecular forces of attraction, leads to coagulation and crystallization of fullerenes.
This occurs in solvents in which molecules have a high electron-donating ability and therefore form a very thin adsorption layer which is unable to hold the fullerene molecule at a desired distance in order to maintain the energy barrier anticoagulation. They are not capable of dissolving the fullerene because of molecular forces of attraction between molecules of the fullerene are stronger than the interaction of the fullerene molecule and a solvent molecule. Therefore they can only be adsorbed on the crystal surface, but do not transfer fullerene molecules into a solution.

As the confirmation it should be noted that the dielectric constant is a parameter correlated with the amount of free charge carriers in solution, as well as the chemical activity of the solvent molecules. In this case it is necessary to pay attention to the fact that the solubility of C60 in toluene is $3 \mathrm{~g} / \mathrm{I}$ at the dielectric constant of 2.4 ; in ethanol $-0.001 \mathrm{~g} / \mathrm{I}$ at the dielectric constant of 24.3 , and $\mathrm{C} 60$ in water is practically insoluble at its dielectric constant 78 .

In such a manner the hydrated fullerene molecule can exist in the very dilute solutions only. A variety of special-purpose methods of attachment of the water molecules to the fullerene molecules is used to produce such hydrated molecules. Thus, the higher the dielectric constant of the solvent (i.e. solvent molecules reactivity for fullerene), the less fullerite soluble in it.

Experiments have shown $[11,12]$, that on the one hand, reducing the number of the solvent molecules used for the construction of micelles, allows a greater amount of fullerene molecules to dissolve, and on the other hand, this reduces the anticoagulation energy barrier. The state of solution, in which the energy of the molecular forces of attraction of fullerene molecules become predominant over the energy of barrier of anticoagulation, can be called as the coagulation threshold of fullerene molecules.

Returning to the section 4 , it is noted that the throwing of the alcohol in the toluene solution of fullerene molecules alcohol molecules form azeotrop with molecules of adsorbed outer layer and detach them from the micelles. In this way they reduce anticoagulation barrier, allowing the fullerene molecules to coagulate and precipitate. In the case of applying an electric field charged colloidal particles deposit on the electrodes.

Thus, the investigation was the answer to the question, why do not all liquids can dissolve the fullerene molecule. 
Table 2: The Solubility of Fullerenes and the Thickness of Adsorbed Layer in Solutions

\begin{tabular}{|c|c|c|c|c|c|c|}
\hline Solvent & 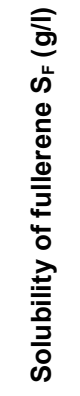 &  & 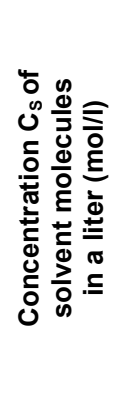 & 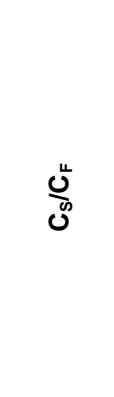 & 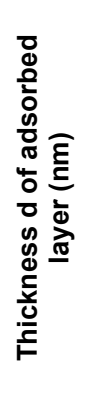 & 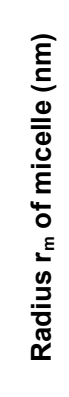 \\
\hline $\begin{array}{c}\text { Carbon disulphide }\left(\mathbf{C S}_{2}\right) \\
\text { M.m.=76,13; } \rho_{4}^{20}=1262,0 \mathrm{~g} / \mathrm{l}\end{array}$ & 7,9 & 0,01083 & 16,577 & 1511 & 2,76 & 3,26 \\
\hline $\begin{array}{l}\text { 1,2-dichlorobenzene }\left(\mathrm{C}_{6} \mathrm{H}_{4} \mathrm{Cl}_{2}\right) \\
\text { M.m. }=147,01 ; \rho_{4}^{20}=1305,3 \mathrm{~g} / \mathrm{l}\end{array}$ & 7,11 & 0,00988 & 8,883 & 899,08 & 2,9 & 3,4 \\
\hline $\begin{array}{c}\text { Chlorobenzene }\left(\mathrm{C}_{6} \mathrm{H}_{5} \mathrm{Cl}\right) \mathrm{M} . \mathrm{m} .=112,55 \\
\rho_{4}^{20}=1106,3 \mathrm{~g} / \mathrm{l}\end{array}$ & 4,57 & 0,00615 & 9,829 & 1590 & 3,2 & 3,7 \\
\hline $\begin{array}{c}\text { Benzene }\left(\mathrm{C}_{6} \mathrm{H}_{6}\right) \\
\text { M.m. }=78,11 ; \rho_{4}^{20}=879,0 \mathrm{~g} / \mathrm{l}\end{array}$ & 1,5 & 0,002083 & 11,254 & 5400 & 5,28 & 5,78 \\
\hline $\begin{array}{c}\text { Pyridine }\left(\mathrm{C}_{5} \mathrm{H}_{5} \mathrm{~N}\right) \\
\text { M.m. }=79,01 ; \rho_{4}^{20}=983,2 \mathrm{~g} / \mathrm{l}\end{array}$ & 0,89 & 0,001235 & 10076,9 & 10078 & 6,3 & 6,8 \\
\hline $\begin{array}{c}\text { Benzonitrile }\left(\mathrm{C}_{6} \mathrm{H}_{5} \mathrm{CN}\right) \\
\text { M.m.=103,08; } \rho_{4}^{20}=1010,2 \mathrm{~g} / \mathrm{l}\end{array}$ & 0,41 & 0,0005689 & 9,8 & 17225 & 8,2 & 8,7 \\
\hline $\begin{array}{l}\text { Dichloromethane }\left(\mathrm{Cl}_{2} \mathrm{CH}_{2}\right) \\
\text { M.m. }=84,91 ; \rho_{4}^{20}=1326,0 \mathrm{~g} / \mathrm{l}\end{array}$ & 0,26 & 0,00036 & 15,617 & 43380 & 9,7 & 10,2 \\
\hline $\begin{array}{c}\text { Tetrahydrofurane - } \\
\text { THF- }\left(\mathrm{CH}_{2}\right)_{4} \mathrm{O} \\
\text { M.m. }=72,1 ; \rho_{4}^{20}=888,3 \mathrm{~g} / \mathrm{l}\end{array}$ & 0,07 & $\begin{array}{c}0,097 \\
10^{-3}\end{array}$ & 12,35 & 127319 & 16,5 & 17 \\
\hline
\end{tabular}

M.m. - molecular mass; $\rho_{4}^{20}$ - density of solvent at $20^{\circ} \mathrm{C}$ relative to water at $40{ }^{\circ} \mathrm{C} ; \mathrm{C}_{\mathrm{S}} / \mathrm{C}_{\mathrm{F}}-$ ratio of the amount of solvent molecules to the amount of fullerene molecules in the saturated solution $(\mathrm{g} / \mathrm{l})$.

\section{The Electron-Donor Strength of the Solvent Molecules for Fullerene in Colloidal II-Type Solutions}

At constant thermodynamic conditions a fullerene molecule is stable [12] and has a stable electron deficiency $(F)$, which determines the number of molecules of the solvent $(X)$ for its compensation. If fullerene molecule is taken as "reference" and taking its electron-deficiency as a unit of measurement if (one fullerene), the electron-donor force $\left(F_{E D}\right)$ of solvent molecule by fullerene will be equal to the ratio of these two values:

$\mathrm{F}_{\mathrm{ED}}=\mathrm{F} / \mathrm{X}$
For different solvents the number of molecules necessary to compensate electron deficiency $(F)$, will be different according to the relationship

$X=F / F_{E D}$

Since the thickness of the adsorbed layer is a function of the number of solvent molecules contained in one micelle, it should be noted that the greater is the electron-donor force $\left(F_{E D}\right)$ of the solvent molecule by fullerene, the less is solvent molecules necessary for compensation of the electron-deficiency $(F)$ of fullerene molecules and the thinner is the adsorption layer and the stronger the intermolecular forces between the molecules of fullerene are. An electron-donating 
strength $\left(F_{E D}\right)$ is a fractional value, which is equal to the ratio for toluene

$F_{E D}=1 \mathrm{f} / \mathrm{X}=1 \mathrm{f} / 2500$

Moreover, by analyzing the ratio between the number of molecules of the solvent and the fullerene molecules (Table 2), it should be emphasized that the amount of the solvent molecules in various solvents is close to the Fibonacci numerical sequence.

This means that the structure of the micelles has a fractal character. Fibonacci series describes the continuous structure of the micelles, and the numbers of this numerical series reflect the special states in the formation of micelle.

\section{Colloidal Solution II-Type}

As we have shown, the colloidal fullerene particle is formed as a charge-transfer complex (CTC). On the formation of the CTC the thermal effect of the dissolution process and the color of the solution indicate. The Fibonacci sequence in the number of solvent molecules per molecule of fullerene, confirms the model of fractal clusters in the micelle structure. This suggests that the fractals in the micelle can be formed as a result of mesomeric or hyper conjugation nature of the interaction between the solvent molecules.

The following features show the formation of fullerene-toluene systems (micelles):

- $\quad$ Presence the surface of the fullerene molecule is a key parameter which allows transferring a molecule of C60 fullerene into the category of colloidal particles;

- $\quad$ Concentration hysteresis on the curve section $A B$ (Figure 2) occurs with decreasing temperature and, presumably, with a decrease in the chemical activity of fullerene molecules, which manifest themselves in violation of the fullerene-toluene complexes;

- Electrophoresis can be explained by the fact that, since ethanol is not reacted with fullerene molecules, then adding ethanol to a solution, alcohol molecules form azeotrop with molecules of toluene, partially destroying shell of the micelles.

For this reason, the complex loses electroneutrality, charges negatively, and is decomposed on the positive electrode, forming a fullerene.
Salting out at low temperatures leads to a more ordered lattice with lower solvate content, than at higher temperatures.

- $\quad$ Photopolymerization (light curing): Experiments have demonstrated that fullerene molecules are present in solution as a CTC to the time of irradiation.

The dualism of fullerene molecule enables it to form during dissolution both the true or molecular-ion solutions and the colloidal but the colloid particle has capability to form the micelles.

The calculations have shown that the energy state of solution, in which the energy of the molecular forces of attraction of fullerene molecules become predominant over the energy of anticoagulation barrier, can be called the coagulation threshold of fullerene molecules.

From the above-mentioned we can make the inference that the fullerene molecule in the $\mathrm{y}$-state exhibits the properties of the colloidal particles and forms the colloidal micellar solution i.e. the colloidal solution of II-type.

\section{CONCLUSIONS}

In this paper it has been revealed that the fullerene molecule in solutions at temperatures above $250 \mathrm{~K}$ exists in two states - $\beta$ and $\gamma$. In this case the dualism of the fullerene molecule allows forming the colloidal solutions in the $y$-state. The existence of the electroneutral fullerene-toluene complexes in solutions has been confirmed experimentally. The fact that in fullerene-toluene complexes the fullerene molecule binds a certain number of solvent molecules, which are determined by their donor activity, have been substantiated experimentally.

We have found that in the colloidal solutions of fullerenes the solvent molecules serve as stabilizers. In this paper we have received an answer to the question why not all liquids can dissolve the fullerene molecules.

The state diagram of fullerene molecules in solution at temperatures of $265-308 \mathrm{~K}$ and in the pressure range of $0.1-100 \mathrm{MPa}$ has been also proposed in this paper.

Fullerene molecule in solutions forms complexes with the charge transfer at temperatures above $270 \mathrm{~K}$. It is stable enough under constant conditions of 
existence and is the reference molecule for determining the donor activity of molecules of various solvents.

It has been shown that the fullerene molecules in solutions at temperatures above $250 \mathrm{~K}$ can form the solutions of three types: the true solution; the colloidal solution of I-type; the colloidal solution of II-type. The colloidal particles in the colloidal solutions of I-type are multimolecular, whereas in the colloidal solution of IItype these particles are monomolecular.

We have found that in the solutions of fullerenes C60 in the $\mathrm{y}$-state the solubility decreases with a rise in temperature since the chemical activity of fullerene molecule increases. For this reason at the extraction the temperature rise causes the value of extraction rate to increase, but solubility - to decrease, i.e. to diminish the concentration of fullerenes molecules in the solvent volume.

Hence, the dualism of fullerene molecule implies that it being a molecule behaves in the $\mathrm{y}$-state as the colloidal particle that allows forming both the true molecular-ion and colloidal solutions in the process of dissolution.

\section{REFERENCES}

[1] Kornilov MYu, Ljubchuk TV, Isaev SD, Panasyuk $\mathrm{H}$, Chernogorenko VB, Schur DV. Perhydrofullerenes as accumulators of hydrogen. Molecular Materials 2000; 13: 285-90.

[2] Yu M Shulga, Tarasov BP, Koshyk D, Drahan V, Zaginaichenko $S \mathrm{Yu}$. Hydride method of $\mathrm{C}_{60}$ fullerite production with "enlarged" fcc lattice. In: Proceed. Int. Symp. "Metal-Hydrogen Systems", Australia, Queensland, Noosa. p. 196. 2000.

[3] Alekseyev NI, Dyuzhev GA. Fullerene formation in an arc discharge. Carbon 2003; 41: 1343-8. https://doi.org/10.1016/S0008-6223(03)00058-7

[4] Schur DV, Zaginaichenko S Yu, Veziroglu TN. Peculiarities of hydrogenation of pentatomic carbon molecules in the frame of fullerene molecule $\mathrm{C}_{60}$. Int $\mathrm{J}$ Hydrogen Energy 2008; 33 : 3330-45.

https://doi.org/10.1016/j.ijhydene.2008.03.064

[5] Schur DV, Zaginaichenko SYu, Lysenko EA, et al. The forming peculiarities of $\mathrm{C}_{60}$ molecule. In: Carbon Nanomaterials in Clean Energy Hydrogen Systems. Dordrecht, Netherlands: Springer. pp. 53-66, 2008.

[6] Schur DV, Matysina ZA, Zaginaichenko S Yu. Carbon nanomaterials and phase transformations in them. Monograph. Dnepropetrovsk, Ukraine: Science and education. 678 p, 2007. (in Russ.)

[7] Schur DV, Zaginaichenko S Yu, Veziroglu TN, Javadov NF. The peculiarities of hydrogenation of fullerene molecules $\mathrm{C}_{60}$ and their transformation (chapter 17). In: Proc. of NATO ARW on the Black Sea: Strategy for Addressing its Energy Resource Development and Hydrogen Energy Problems. Dordrecht, Netherlands: Springer. pp. 191-204, 2013. https://doi.org/10.1007/978-94-007-6152-0 17
Anikina NS, Schur DV, Zaginaichenko S Yu, et al. Regularity of fullerene $\mathrm{C}_{60}$ dissolution in polymethyl-substituted benzenes. Nanosystems, Nanomaterials, Nanotechnologies 2013; 11: 173-92 (in Russ.).

[9] Schur DV, Zaginaichenko S Yu, Veziroğlu TN. The hydrogenation process as a method of investigation of fullerene $\mathrm{C}_{60}$ molecule. Int $\mathrm{J}$ Hydrogen Energy 2015; 40: 2742-62.

https://doi.org/10.1016/j.ijhydene.2014.12.092

[10] Schur DV. The physico-chemical regularities of synthesis extraction, crystallization processes and application of fullerene $\mathrm{C}_{60}$. Manuscript. Thesis of Dr. chem. sci. diss. Kiev, Ukraine. 336 p., 2014 (in Russ.)

[11] Schur DV, Zaginaichenko S Yu, Shulga Yu M, Anikina NS, Polyschuk M.A. Study of features of the salting-out process of fullerite from saturated solution of $\mathrm{C}_{60}$ in toluene. I. Laminar mixing of reagents. Nanosystems, Nanomaterials, Nanotechnologies 2013; 11: 815-32 (in Russ.).

[12] Schur DV, Zaginaichenko S Yu, Kotko AV, Anikina NS Kamenetskaia E.A. Study of features of the salting-out process of fullerene from saturated solution of $\mathrm{C}_{60}$ in toluene. II. Turbulent mixing of reagents. Nanosystems, Nanomaterials, Nanotechnologies 2013; 11: 832-61 (in Russ.).

[13] Anikina NS, Krivuschenko O Ya, Mil'to OV, Zolotarenko EP Schur DV, Zaginaichenko S Yu. The interaction of soluble carbon nanostructures with aromatic solvents. Nanosystems, Nanomaterials, Nanotechnologies 2013; 11: 193-216 (in Russ.).

[14] Anikina NS, Krivuschenko O Ya, Schur DV, Zaginaichenko S $\mathrm{Yu}$, Kamenetskaia EA. Special features and regularities of interaction between fullerene molecules and aromatic solvents (chapter 4). In: Carbon Nanomaterials in Clean Energy Hydrogen Systems. Dordrecht, Netherlands: Springer. pp. 53-74, 2011 https://doi.org/10.1007/978-1-4020-5514-0 2

[15] Anikina NS, Schu, DV, Zaginaichenko S Yu, Krivuschenko O $\mathrm{Ya}$, Kale A. Investigation of mechanism of fullerene dissolution in aromatical hydrocarbons. In: Hydrogen Materials Science and Chemistry of Carbon Nanomaterials. Dordrecht, Netherlands: Springer. pp. 19-36, 2007.

[16] Ruoff RS, Malhotra R, Huestis DL, Tse DS, Lorents DC Anomalous solubility behavior of $\mathrm{C}_{60}$. Nature $1993 ; 362$ : 140 1. https://doi.org/10.1038/362140a0

[17] Sawamura S, Fujita N. High-pressure solubility of fullerene $\mathrm{C}_{60}$ in toluene. Carbon 2007; 45: 965-70. https://doi.org/10.1016/j.carbon.2006.12.030

[18] Matysina ZA, Zaginaichenko S Yu, Schur DV, et al. $\mathrm{C}_{60}$ fullerite solubility in organic matrix. Nanosystems, Nanomaterials, Nanotechnologies 2009; 7: 671-81 (in Russ.).

[19] Matysina ZA, Zaginaichenko S Yu, Schur DV. The solidphase transformations in fullerite stimulated by rearrangement of structure of fullerene molecules. Metal Physics and Advanced Technologies 2009; 31: 987-1000 (in Russ.).

[20] Khotynenko NG, Schur DV, Zaginaichenko S Yu, et al. On electrochemical deposition of fullerenes and their compounds from solutions. In: Hydrogen Materials Science and Chemistry of Carbon Nanomaterials. Dordrecht, Netherlands: Springer. pp. 287-299, 2007. https://doi.org/10.1007/978-1-4020-5514-0 38

[21] Wang Y, Holden JM, Bi XX, Eklund PC. Thermal decomposition of polymeric $\mathrm{C}_{60}$. Chem Phys Lett 1994; 217: 413-7. https://doi.org/10.1016/0009-2614(93)E1409-A

[22] Eklund PC, Rao AM, Zhou P, Wang $Y$, Holden JM. Photochemical transformation of $\mathrm{C}_{60}$ and $\mathrm{C}_{70}$ films. Thin Sol Films 1995; 257: 185-203.

https://doi.org/10.1016/0040-6090(94)05704-4 
[23] Pusztai T, Oszlanyi G, Faigel G, et al. Bulk structure of phototransformed C60. Sol St Commun 1999; 111: 595-9. https://doi.org/10.1016/S0038-1098(99)00267-7
[24] Zaginaichenko S Yu, Matysina ZA, Schur DV. The statistical theory of photo- and electropolymerization of fullerenes. Nanosystems, Nanomaterials, Nanotechnologies 2012; 10: $731-45$ (in Russ.). 